\title{
Recursive families of OWA operators
}

\author{
Vincenzo CUTELLO \\ Department of Mathematics \\ University of Catania \\ Catania, Italy
}

\author{
Javier MONTERO \\ Department of Statistics and O.R. \\ Complutense University \\ Madrid, Spain
}

\begin{abstract}
In this paper we introduce an alternative characterization of OWA operators by means of recursive definitions on their dimension. Some mathematical results will be provided along with some examples concerning also a characterization of linguistic quantifiers by means of these recursive definitions of OWA operators.
\end{abstract}

Key words: Aggregation rules, fuzzy sets, linguistic quantifiers.

\section{INTRODUCTION AND Preliminaries}

Intelligent aggregation of information and related complexity issues represent central problems in any decisional process. Usually, information is passed to an aggregation operator as an ordered sequence of real numbers, which without loss of generality can be supposed to belong to the unit interval. Such a simple hypothesis is still sufficiently general to cover a wide set of applications.

First examples of aggregation operators are $\mathrm{T}$ norms and $\mathrm{T}$-conorms which generalize the notion of conjunction and disjunction of classical logic. The min operator is the maximal T-norm and the $\max$ operator is the minimal T-conorm (see [6].) To fil1 the gap between min and max, Ordered Weighted Averaging ( $O W A$ ) operators were proposed by Yager in [9] and more recently characterized in [10].

It is clear that there are considerably many real life decision processes where at different times one has to aggregate (possibly very large) lists of inputs of different dimensions. In this context, we can identify two main problems:

1. computational problems of aggregating large lists of inputs;

2. semantic problems of using the same aggregation operator everytime this is the case, on inputs of different dimensions.

In order to improve the computational complexity of the aggregation process, a theoretical framework of hierarchical aggregations -for general aggregation operators as well as OWA operators- was proposed and characterized in $[2,3,4]$.

In this paper we shall introduce a formal method for a recursive definition of OWA operators. Such a recursive definition will depend upon the number of values already aggregated. A particular case appears when we do not have to specify such a number, i.e. our recursive definition is independent from the size of the inputs itself. This is the case for the two limit OWA operators, namely $\min$ and $\max$, and more generally for T-norms and T-conorms. Indeed, $\mathrm{T}$-norms and $\mathrm{T}$-conorms are associative. Thus, given any $\mathrm{T}$-norm or T-conorm $F(x, y)$ and given any $n$ values $\left(a_{1}, \ldots, a_{n}\right)$, we can apply the same operative definition of $F(x, y)$ to obtain $F\left(a_{1}, \ldots, a_{n}\right)$. More in details, we see that we can evaluate each 
value $F\left(a_{1}, \ldots, a_{n}\right)$ either as $F\left(F\left(a_{1}, \ldots, a_{n-1}\right), a_{n}\right)$ or $F\left(a_{1}, F\left(a_{2}, \ldots, a_{n}\right)\right)$. In the first case the final value is obtained by means of a left recursion call and in the second case by means of a right recursion call to the same operative procedure. Although such a procedure can not be considered when dealing with OWA operators, a recursive analysis of OWA opeartors can be applied to the ordered list associated to $\left(a_{1}, \ldots, a_{n}\right)$.

Moreover, this approach suggets a natural way of defining families of OWA operators: those OWA operators defined by means of a certain sequence that achieves the information about the number of values to be aggregated (dependence on the number of aggregated values already appeared in $[7,8]$ as a key assumption in order to avoid Fung-Fu's theorem [5] in group decision making). Thus, practical aggregation problems, where the number of values to be aggregated is not necessarely previously known, should be solved by choosing one of these families of OWA operators, in such a way that each one of these families solves every aggregation problem for any arbitrary size of the input. Moreover, our recursive definition has also the advantage that aggregation weights can be computed quickly by using a dynamic programming approach (see [1]).

\section{Formal DEFINITION OF OWA OPERATORS}

To simplify the formalization of OWA operators let us introduce the notion of sorting permutation of a list.

If $L=\left[a_{1}, a_{2}, \ldots, a_{n}\right]$ is a list of numbers, a sorting permutation $\sigma$ for $L$ is any permutation of the elements of $L$ that produces a list $\sigma(L)=\left[a_{[1]}, \ldots, a_{[n]}\right]$ verifying $a_{[i]} \geq a_{[j]}$ for all $i \leq j$.

DEFINITION 1 An OWA operator of dimension $n$ is an aggregation operator $\phi$ that has an associated list of weights $W=\left[w_{1}, \ldots, w_{n}\right]$ such that

1. $w_{i} \in[0,1]$ for all $1 \leq i \leq n$

2. $\sum_{i=1}^{n} w_{i}=1$
3. for any $L=\left[a_{1}, a_{1}, \ldots, a_{n}\right]$ and its corresponding $\sigma(L)=\left[a_{[1]}, \ldots, a_{[n]}\right]$

$$
\phi(L)=\sum_{i=1}^{n} w_{i} a_{[i]} .
$$

In view of the above definition, it can be immediately verified that $O W A$ operators are commutative, monotone and idempotent, but in general not associative.

A significative measure associated with OWA operators is the orness which estimates how close an OWA operator is to the max operator. It is defined as

$$
\operatorname{orness}(\phi)=\frac{1}{n-1} \sum_{i=1}^{n}(n-i) w_{i} .
$$

Dual to the measure of orness is the measure of andness defined as andness $(\phi)=1-$ orness $(\phi)$, which therefore measures how close an OWA operator is to the min operator.

An important notion is duality. Given an OWA operator $\phi$ with weights $\left[w_{1}, \ldots, w_{n}\right]$, the dual $\hat{\phi}$ of $\phi$ is the OWA operator whose weights are $\left[w_{n}, \ldots, w_{1}\right]$. It is not difficult to see that orness $(\hat{\phi})=\operatorname{andness}(\phi)$.

Finally, a particular class of OWA operator is given by the buoyancy measures. They are OWA operators that verify the property $w_{i} \geq w_{j}$ if $i<j$. Any buoyancy measure $\phi$ is such that orness $(\phi) \geq \frac{1}{2}$.

\section{RECURSIVE DEFINITION OF OWA OPERATORS}

Every OWA operator can be recursively defined, both left and right, once the $n$ values to be aggregated have been ordered. Moreover, these two recursive representations are unique, as shown in the following result.

THEOREM 1 Let us consider a fixed OWA operator $\phi$ of dimension $n$. Then there exist a unique family of $n-1$ OWA operators of dimension $2, F_{2}, \ldots, F_{n}$, and another unique family of $n-1 O W A$ operators all of them also of dimension $2, G_{1}, G_{2}, \ldots, G_{n}$, allowing 
a left recursion and a right recursion, respectively, in such a way that $\phi\left(a_{1}, \ldots, a_{n}\right)$ is equivalent to

$$
\begin{aligned}
& \quad F_{n}\left(\ldots, F_{3}\left(F_{2}\left(a_{[1]}, a_{[2]}\right), a_{[3]}\right), \ldots, a_{[n]}\right) \\
& \text { and } \\
& G_{n}\left(a_{[1]}, \ldots,\left(a_{[n-3]}, G_{3}\left(a_{[n-2]}, G_{2}\left(a_{[n-1]}, a_{[n]}\right)\right)\right)\right)
\end{aligned}
$$

\section{FAMILIES OF RECURSIVELY DEFINED OWA OPERATORS}

We will now introduce our main definition. A basis function is any mapping $f$ that to any integer $n$ associates a number in the unit interval (that is, $f(n) \in[0,1]$ for all $n$ ) with $f(1)=1$. Each basis function $f$ will allow the recursive definition of two families of OWA operators: for any $n \geq 2$, let us denote by $F_{n}$ and $F_{n}^{\prime}$ the two-dimension OWA operators such that

$$
F_{n}\left(b_{1}, b_{2}\right)=(1-f(n)) b_{[1]}+f(n) b_{[2]}
$$

and

$$
F_{n}^{\prime}\left(b_{1}, b_{2}\right)=f(n) b_{[1]}+(1-f(n)) b_{[2]}
$$

Then any left recursive operation

$$
F_{n+1}\left(F_{n}\left(\ldots\left(F_{2}\left(a_{[1]}, a_{[2]}\right)\right), \ldots, a_{[n]}\right), a_{[n+1]}\right)
$$

and any right recursive operation

$$
F_{n+1}^{\prime}\left(a_{[1]},\left(F_{n}^{\prime}\left(a_{[2]}, \ldots, F_{2}^{\prime}\left(a_{[n]}, a_{[n+1]}\right)\right)\right)\right)
$$

will always lead to OWA operators, for every $n \geq 2$, as it will be shown below. Each one of these two families of OWA operators $\left(\Phi=\left\{\phi_{2}, \ldots, \phi_{n}, \ldots\right\}\right.$ if obtained via LR and $\Phi^{\prime}=\left\{\phi_{2}^{\prime}, \ldots, \phi_{n}^{\prime}, \ldots\right\}$ if obtained via RR), will be then associated to the basis function $f$. LR and RR families of OWA operators will be defined, in particular, as follows:

- $n$ is the dimension of the OWA operators $\phi_{n}$ and $\phi_{n}^{\prime}$;

- the weights of $\phi_{n}$ are denoted by $w_{1, n}, \ldots, w_{n, n}$ and $w_{1, n}^{\prime}, \ldots, w_{n, n}^{\prime}$ will denote the weights of $\phi_{n}^{\prime}$;
- for every $n \geq 2$ and every $i=1,2, \ldots, n$ we define

$$
w_{i, n}= \begin{cases}f(n) & \text { if } i=n \\ (1-f(n)) w_{i, n-1} & \text { if } i<n\end{cases}
$$

- for every $n \geq 2$ and every $i=1,2, \ldots, n$ we define

$$
w_{i, n}^{\prime}= \begin{cases}f(n) & \text { if } i=1 \\ (1-f(n)) w_{i-1, n-1}^{\prime} & \text { if } i>1\end{cases}
$$

Therefore,

- $w_{i, n}=f(i) \prod_{j=i+1}^{n}(1-f(j))$ for every $n \geq 2$ and every $i=1,2, \ldots, n$.

- $w_{i, n}^{\prime}=f(n-i+1) \prod_{j=n-i+2}^{n}(1-f(j))$ for every $n \geq 2$ and every $i=1,2, \ldots, n$.

In view of the above equations it is immediate to check that $\phi_{n}$ and $\phi_{n}^{\prime}$ are in fact OWA operators, since for any $n \geq 2$ we have

$$
\sum_{i=1}^{n} w_{n, i}=\sum_{i=1}^{n} w_{n, i}^{\prime}=1
$$

Obviously, not every family $\left\{\phi_{2}, \ldots, \phi_{n}, \ldots\right\}$ of OWA operators can be recursively defined as above, by means of OWA operators of dimension 2. For example, if $w_{i, k}=0$, in order to be able to provide a left recursive characterization it must also be $w_{i, k+1}=0$. Analogously, $w_{i+1, k+1}=0$ must hold for a right recursive definition, whenever $w_{i, k}=0$.

From theorem 1 it is implied that $\phi_{n}$ of dimension $n$ being fixed, then all LR (and RR) consistent OWA operators with lower dimension $\phi_{2}, \ldots, \phi_{n-1}$ are univocally defined. More in general, we have the following result, which also gives the formal definition of such a consistency.

THEOREM 2 Let us consider a family of $O W A$ operators $\left\{\phi_{2}, \ldots, \phi_{n}, \ldots\right\}$. Then it can be defined by $L R$ (i.e., it is LR consistent) if and only if $w_{i, k} w_{j, k+1}=w_{j, k} w_{i, k+1}$ for all $i, j=1,2, \ldots, k$ and every $k$. Anlogously, such a family of $O W A$ operators can be defined by $R R$ (i.e., it is RR consistent) if and only if $w_{i, k} w_{j+1, k+1}=w_{j, k} w_{i+1, k+1}$ for all $i, j=1,2, \ldots, k$ and every $k$. 
Therefore, once any $\phi_{n}$ has been chosen, clear restrictions are implied in order to obtain families of OWA operators which are consistent with $\phi_{n}$, both with respect to left recursion and right recursion. But if a left (right) recursion exists, the associated LR (R$R$ ) basis function is unique. Thus, each basis function is characterizing a LR (RR) consistent family of OWA operators. Moreover, it must be also pointed out that the existence of a right (left) recursion representation for a given family $\Phi=\left\{\phi_{2}, \ldots, \phi_{n}, \ldots\right\}$ of OWA operators does not imply the existence of a left (right) recursion representation. For example, $\phi_{2}$ such that $w_{1,2}=w_{2,2}=1 / 2$ and $\phi_{3}$ such that $w_{1,3}=w_{2,3}=1 / 4$ and $w_{3,3}=1 / 2$ are consistent with respect to left recursion, but not with respect right recursion.

Hence, an interesting case to be analyzed will be the one in which both left and right recursion$s$ do exist for a given family of OWA operators $\left\{\phi_{2}, \ldots, \phi_{n}, \ldots\right\}$, being $f$ and $g$ its LR and RR basis functions, in such a way that

$$
\begin{aligned}
& F_{n+1}\left(F_{n}\left(\ldots\left(F_{2}\left(a_{[1]}, a_{[2]}\right)\right), \ldots, a_{[n]}\right), a_{[n+1]}\right)= \\
& \quad=G_{n+1}^{\prime}\left(a_{[1]},\left(G_{n}^{\prime}\left(a_{[2]}, \ldots, G_{2}^{\prime}\left(a_{[n]}, a_{[n+1]}\right)\right)\right)\right)
\end{aligned}
$$

for any $n$ and any $\left[a_{1}, \ldots, a_{n}\right]$. The associated $f$ and $g$ will be here the basis functions of the left and right recursion, respectively, defining the same family of OWA operators $\left\{\phi_{2}, \ldots, \phi_{n}, \ldots\right\}$. Most usual families of OWA opeartors do belong to this class, as shown in the next section.

The following theorem refers to duality, and the next one can be quite useful in estimating the orness of recursive OWA operators.

THEOREM 3 Let $f$ be a basis function with associated LR family of OWA operators $\Phi$, and $\Phi^{\prime}$ its associated $R R$ family. Then $\hat{\phi}_{n}^{\prime} \equiv \hat{\phi}_{n}$ for all n, i.e. $\phi_{n}^{\prime}$ is the dual of $\phi_{n}$ for all $n$.

THEOREM 4 Let $f$ be basis function such that $(1-f(n)) f(n-1) \geq f(n)$ for all $n$, and let us denote by $\Phi$ and $\Phi^{\prime}$ the corresponding $L R$ and $R R$ families of $O W A$ operators, respectively. Then every $\phi_{n}$ is a buoyancy measure. Therefore, orness $\left(\phi_{n}\right) \geq \frac{1}{2}$ and $\operatorname{andness}\left(\phi_{n}^{\prime}\right) \geq \frac{1}{2}$.

\section{EXAMPLES}

We will now provide some interesting examples of recursive families of OWA operators.

\section{A. Mean average}

The following result characterizes the mean rule.

THEOREM 5 Let $f$ be a basis function with associated $L R$ and $R R$ families of $O W A$ operators being identical $\left(\Phi \equiv \Phi^{\prime}\right.$, that is, $\phi_{n} \equiv \hat{\phi_{n}}$ for all $\left.n\right)$. Then it must be $f(n)=1 / n$ for all $n$, and in turn the weights of each $\phi_{n}$ are $w_{i, n}=1 / n$ for all $i=1,2, \ldots, n$.

\section{B. Constant basis function}

A special case will indeed appear when there exists a value $a \in[0,1]$ such that $f(n)=a$ for all $n \geq 2$. Hence, each LR OWA operator $\phi_{n}, n \neq 2$ will have weights $w_{1, n}=(1-a)^{n-1}$, and $w_{i, n}=(1-a)^{n-i} a$ for all $i=2, \ldots, n$. Analogously, weights for each RR OWA operator $\phi_{n}^{\prime}$ will be $w_{i, n}=(1-a)^{i-1} a$ for all $i=1,2, \ldots, n-1$ and $w_{n, n}=(1-a)^{n-1}$.

When $a=1(a=0)$ in left (right) recursion we obtain the family of min operators, and in right (left) recursion we obtain its dual family of $\max$ operators.

\section{Harmonic OWA operators}

We recall that the $n$-th harmonic number is $H_{n}=$ $\sum_{i=1}^{n} \frac{1}{i}$. Harmonic OWA operators are obtained by taking $f(n)=\frac{1}{H_{n}}$. Thus, $1-f(n)=\frac{H_{n-1}}{H_{n}}$.

By using theorem 4 it is immediate to see that the family of $L R$ - Harmonic OWA operators is a class of buoyancy measures. For example, its first OWA operators will have the following weights:

$$
\begin{array}{r}
w_{1,2}=\frac{2}{3}, w_{2,2}=\frac{1}{3} \\
w_{1,3}=\frac{6}{11}, w_{2,3}=\frac{3}{11}, w_{3,3}=\frac{2}{11} \\
w_{1,4}=\frac{12}{25}, w_{2,4}=\frac{6}{25}, w_{3,4}=\frac{4}{25}, w_{4,4}=\frac{3}{25}
\end{array}
$$

D. A monotone fuzzy quantifier

In $[9,10]$ it is shown how to obtain the evaluation of monotone fuzzy quantifiers by means of OWA operators. In particular, given a monotone non decreasing 
fuzzy quantifier $Q$ such that $Q(0)=0$ and $Q(1)=1$, the weights $w_{i, n}$ for $i=1,2, \ldots, n$ of an OWA operator of dimension $n$ to evaluate $Q$ are defined as

$$
w_{i, n}=Q\left(\frac{i}{n}\right)-Q\left(\frac{i-1}{n}\right)
$$

In case $Q(a)=a^{r}$ for some $r>0$ we obtain that taking $f(n)=1-Q\left(\frac{n-1}{n}\right)$ for all $n$, the associated left-recursive family of OWA operators does verify such a property. Hence, such a monotone fuzzy quantifier allows a left recursive definition. But it can not be right-recursively defined.

\section{Final Comments}

In this paper, we introduced the notion of recursive families of OWA operators. We provided in particular a theoretical framework for constructing such families either by means of left or right recursion calls. Some results are also given concerning dual operators and the measure of orness. Finally, we have shown several examples of recursive families of OWA operators. It can therefore be suggested that many practical applications of OWA operators should be developed by defining a (LR or RR) consistent family of OWA operators, allowing an efficient procedure when the number of values to be aggregated is not previously known.

Acknowledgment: This research has been partially supported by Dirección General de Investigación Científica y Técnica (Spanish national grant PB91-0389).

\section{REFERENCES}

[1] T.H. Cormen, C.E. Leiserson and R.R. Rivest Introduction to Algorithms. MIT Press, Cambridge, MA, 1990.

[2] V. Cutello and J. Montero. Hierarchies of intensity preference aggregations. International Journal of Approximate Reasoning, 1993. To appear.
[3] V. Cutello and J. Montero. Hierarchies of aggregation operators. International Journal of Intelligent Systems, 1993. To appear.

[4] V. Cutello and J. Montero. Computational problems of the hierarchical aggregation of OWA operators. 1993. Submitted.

[5] L.W. Fung and K.S. Fu. An axiomatic approach to rational decision making in a fuzzy environment. In L.A. Zadeh, K.S. Fu, K. Tanaka, and M. Shimura, editors, Fuzzy sets and their applications to Cognitive and decision processes, pages 227-256. Academic Press, 1975.

[6] G.J. Klir and T.A. Folger. Fuzzy sets, Uncertainty and Information. Prentice Hall, Englewood Cliffs, NJ, 1988.

[7] J. Montero. A note on Fung-Fu's theorem. Fuzzy sets and systems, 17:259-269, 1985.

[8] J. Montero. Aggregation of fuzzy opinions in a non-homogeneous group. Fuzzy sets and systems, 25:15-20, 1988.

[9] R.R. Yager. On ordered weighted averaging aggregation operators in multi-criteria decision making. IEEE transactions on Systems, Man and Cybernetics, 18:183-190, 1988.

[10] R.R. Yager. Families of OWA operators. Fuzzy sets and systems, 59:125-148, 1993. 